\title{
Epidemiological Factors in Patients with Dermatologic Conditions Referring to Clinic of Traditional Persian Medicine, Shiraz, Southern Iran during 2018: A Cross- Sectional Study
}

\author{
Mohammad Mahdi Parvizi ${ }^{1}$, Nazanin Fatehi ${ }^{1}$, Amir Mohammad Jaladat ${ }^{1}$, Zahra \\ Gholampour $^{1}$, Reza Shahriarirad ${ }^{2}$, and Amirhossein Erfani ${ }^{2}$ \\ ${ }^{1}$ Shiraz University of Medical Sciences \\ ${ }^{2}$ Affiliation not available
}

January 13, 2021

\begin{abstract}
Introduction: Many patients with chronic diseases often use the traditional medicine approach in addition to conventional medicine. The aim of this study was to evaluate the epidemiological factors of patients with dermatologcal conditions referring to traditional Persian Medicine (TPM) clinic. Materials and Methods: In this cross-sectional study, we reviewed all the patients' medical documents with dermatological conditions referred to the TPM clinic at Shiraz, Southern Iran in 2018. Then, the epidemiological factors of the patients were recorded to a researcher-made data collection form. SPSS software version 22 was conducted to data analysis. Results: Overall, 631 patients consist of 151 (23.9\%) male individuals and 580 (76.1\%) female individuals were enrolled to the study. Most of the patients were aged between 31 and 40 years, and 347 (55\%) patients were married, and most of them were housewives. The frequency of patients in spring was higher than other seasons. Furthermore, the most of the patients were university educated. Acne, eczema and hair loss were the three most complaints of the patients referring to the traditional medicine clinic. 301(47.7\%) patients reported gastrointestinal problems as another complaint concomitant with their skin problems. Conclusion: It seems that women with skin problems, including acne, eczema, and hair loss, were more likely to refer to the TPM clinic. Therefore, development of TPM clinics in dermatology field as a scientific and academic approach can be effective in the treatment of patients with dermatological condition accompanied by traditional medicine.
\end{abstract}

Title page

Type of manuscript: Original Article

Title: Epidemiological Factors in Patients with Dermatologic Conditions Referring to Clinic of Traditional Persian Medicine, Shiraz, Southern Iran during 2018: A Cross- Sectional Study

Short running title: Dermatologic ptients referring to TPM clinics

Mohammad Mahdi Parvizi ${ }^{1}$, Nazanin Fatehi $^{2}$, Amir Mohammad Jaladat ${ }^{3}$, Zahra Gholampour $^{4}$, Reza Shahriarirad ${ }^{2,5}$, Amirhossein Erfani ${ }^{2,5}$

${ }^{1}$ Molecular Dermatology Research Center, Shiraz University of Medical Sciences, Shiraz, Iran

${ }^{2}$ Student Research Committee, Shiraz University of Medical Sciences, Shiraz, Iran

${ }^{3}$ Department of Traditional Persian Medicine, Shiraz University of Medical Sciences, Shiraz, Iran.

${ }^{4}$ Hakim Emad-al-Din Traditional Persian Medicine Clinic, Shiraz University of Medical Sciences, Shiraz, Iran. 
${ }^{5}$ Thoracic and Vascular Surgery Research Center, Shiraz University of Medical Sciences, Shiraz, Iran

The corresponding authors of this manuscript is:

Mohammad Mahdi Parvizi, MD, PHD. Dermatology Research Center, Shiraz University of Medical Sciences, Shahid Faghihi Hospital, Shiraz, Iran. ORCID ID: 0000-0003-1856-945X , Telefax: +98-7132319049, Tel: +98-7132315592, Cell phone: 00989173237031, Email: mmparvizi@gmail.com

Amir Mohammad Jaladat: Department of Traditional Persian Medicine, Shiraz University of Medical Sciences, Shiraz, Iran. ORCID ID:0000-0003-3158-1250, Tel: +98-7132349983, Cell phone: 00989173015789, email: drjaladat@gmail.com

\section{Acknowledgment:}

This article was extracted from the thesis written by Nazanin Fatehi as partial fulfillment of the requirements for obtaining her medical degree. Furthermore, this study was supported by Vice Chancellor of Research, Shiraz University of Medical Sciences, and Shiraz, Iran. [Grant number 97-01-01-18002].

Epidemiological Factors in Patients with Dermatologic Conditions Referring to Clinic of Traditional Persian Medicine, Shiraz, Southern Iran during 2018: A Cross- Sectional Study

\section{Abstract}

Introduction: Many patients with chronic diseases often use the traditional medicine approach in addition to conventional medicine. The aim of this study was to evaluate the epidemiological factors of patients with dermatologcal conditions referring to traditional Persian Medicine (TPM) clinic.

Materials and Methods: In this cross-sectional study, we reviewed all the patients' medical documents with dermatological conditions referred to the TPM clinic at Shiraz, Southern Iran in 2018. Then, the epidemiological factors of the patients were recorded to a researcher-made data collection form. SPSS software version 22 was conducted to data analysis.

Results: Overall, 631 patients consist of 151 (23.9\%) male individuals and 580 (76.1\%) female individuals were enrolled to the study. Most of the patients were aged between 31 and 40 years, and $347(55 \%)$ patients were married, and most of them were housewives. The frequency of patients in spring was higher than other seasons. Furthermore, the most of the patients were university educated. Acne, eczema and hair loss were the three most complaints of the patients referring to the traditional medicine clinic. 301(47.7\%) patients reported gastrointestinal problems as another complaint concomitant with their skin problems.

\section{Conclusion:}

It seems that women with skin problems, including acne, eczema, and hair loss, were more likely to refer to the TPM clinic. Therefore, development of TPM clinics in dermatology field as a scientific and academic approach can be effective in the treatment of patients with dermatological condition accompanied by traditional medicine.

Keyword: Skin diseases, Epidemiological factors, Traditional medicine, Iran

What is already known about this topic?According to the literature, the most of patients suffering from chronic diseases, especially patients with chronic dermatologic conditions, use herbal, natural or complementary remedies, even without a doctor's or complementary and traditional medicine practitioner's prescription.

What does this article add?This article reveals that many patients with chronic dermatologic conditions, who were dissatisfied with the results of treatment with conventional medicine, referred to complementary and traditional medicine physicians in an academic tertiary out-patient clinic of this field in Iran to receive prescription for their problems and more than $50 \%$ of them have experienced moderate to good therapeutic responses. 


\section{Introduction}

Dermatoogy conditions are one of the most common human diseases, which affect $30 \%$ - $70 \%$ of people regardless with individual cultures and ages The International Classification of Diseases lists over 1,000 dermatologic or skin-related diseases in 10 categories. Internationally, skin diseases are the fourth leading cause of non-fatal diseases. Despite this abundance, skin diseases continue to receive less attention in national and global health debates (1).

Patients with dermatologic problems often experience emotional problems, such a way that the prevalence of psychiatric disorders among dermatologic patients was reported between $30 \%$ and $60 \%(2-4)$. Chronic skin diseases are considered as long-standing diseases that has had an increasing rate in recent years especially in developed countries. But the mortality rate of dermatology diseases has been decreased because of presenting new medication and better managent the disease by general physicians and dermatologists. In this regard, advances in the treatment of chronic skin diseases have improved the patients' quality of life(5) .

Several skin diseases are associated with pain and discomfort, physical disabilities and psychological disorders $(6,7)$. In some conditions, complications of skin diseases usually remain permanently or temporarily. Therefore, these complications can affect the patients' professional, psychological and physical well-being (8). On the other hand, there is some evidence that suggests the quality of life in patients with dermatological is equal to or worse than the quality of life in patients with cancer or heart diseases (9).

Tendency to use folklore, complementary and integrative medicine is prominent among the Iranian population, especially in patients (10-12). In this regard, many people with chronic disorders, such as several dermatological conditions, have resorted to the use of integrative and complementary medicine, natural and herbal remedies, acupressure, acupuncture, exercise, psychological and nutritional approaches to control the symptoms of the diseases (13-15).

To the best of our knowledge, despite the high number of patients with dermatologic conditions who referred to TPM clinics, the epidemiological studies were not conducted in these patients in Iranian population. In this survey, we decided to assess the epidemiological charastristics in patients with any dermatological conditions referring to TPM clinic affiliated to Shiraz University of Medical Sciences, Shiraz, Southern Iran, during 2018.

\section{Materials and methods}

\section{Study design and population:}

This cross-sectional descriptive study, was conducted in all patients with dermatologic conditions referring to the Hakin Emad-al-Din Shirazi clinics, the central TPM clinic affiliated to Shiraz University of Medical Sciences, Shiraz, Iran during 2018.

\section{Ethics statements:}

Ethics Committee of Shiraz Medical School approved the protocol of this study (Code: IR.SUMS.MED.REC.1398.189). The data collection process was completely confidential and an individual code was assigned to identification of each patient.

\section{Data collection:}

For data collection, the researchers recorded the demographical charastristics of the enrolled patients, including age, sex, marital status, educational level, occupation, season of the visit, nationality, and as well as dermatologic conditions in a researcher-made data collection form. For assessment the patients' response to the treatment we used Patient Global Impression Scale of Improvement (PGI-I). Furthermore, the researchers called the patients to obtain any missing information.

\section{Statistical analysis:}


SPSS software version 22 (IBM Corporation, Armonk, NY) was used to analyze the data. The results of the study were reported as "mean \pm standard deviation" for quantitative variables and as "number (percent)" for qualitative ones. The Chi-square test was used to determine associations between the variables. The significance level of p-value was considered 0.05 or less.

\section{Results:}

Overall, 631 patients with dermatological conditions were enrolled to the study. The patients aged from 6 months to 91 years (mean $=63.45, \mathrm{SD}=32.14$ ) and the patients' disease duration ranged from 8 months to 40 years $($ mean $=6.21, \mathrm{SD}=5.25)$. All of these patients with dermatological complaints were visited and diagnosed by one or more dermatologist before been referred to the TPM clinic. Table 1 demonstrates the epidemiological characteristics of the patients with dermatological conditions referred to TPM clinic were presented in Table 1.

The majority of skin patients referred to the TPM clinic (99.5\%) were Iranian. The place of residence of the majority of skin patients referred to the TPM (77.8\%) was Shiraz. The majority of patients (19.9\%) referred to the traditional medicine clinic in May.

Table 2 demonstrates the primary dermatological complaints of patients referring to TPM clinic. The majority of primary skin complaints of the patients $(27.6 \%)$ consisted of acne and the least for hirsutism and premature graying of hair $(0.3 \%)$.

Based on our study, 148 out of 631 patients(23.5\%) suffered from only dermatological complaints. However, 163 patients $(25.8 \%)$ suffered from another comorbid complaint, in addition to their skin conditions and 320 patients $(50.7 \%)$ suffered from two comorbid problems. In this setting, most patients $(47.7 \%)$ had gastrointestinal problems in addition to their initial skin complaints. Table 2 describes the comorbidities documented in the patients in our study.

In the follow-up course of the patients under treatment with TPM, we could follow up 271 out of 631 patients. The result of following up these patients demonstrated that 124 (45.8\%) of them had reported less than $30 \%$ improvement, 29 (10.7\%) had announced 30\%-60\% improvement and $118(43.5 \%)$ had experienced a recovery of more than $60 \%$. In addition, there were no associations between the rate of improvement and the patients' sex $(\mathrm{p}=0.430)$, level of education $(\mathrm{p}=0.853)$, place of residence $(\mathrm{p}=0.336)$, and the numbers of visiting the TPM physicians.

\section{Discussion}

Most of patients referring to TPM clinics were suffering from chronic diseases, which had not received appropriate treatment from modern medicine(16). The literature revealed that the most patients who referred to TPM clinics had selected traditional treatment as the end step of their therapeutic process and they hope that they would have had an acceptable therapeutic response to improve the quality of life $(17,18)$.

The findings of the present study showed that the majority of patients referred to TPM clinic were married, because it seems that married people tend to seek treatment and follow-up for their skin condition due to their appearance and cosmetic factors. Also, the education level of the most of the patients with dermatologic problems referring to the TPM clinic was a bachelor's degree. This finding demonestrated that people with a higher degree of education probably had more tendency to try TPM remedies for treatment of their disease which also confirms the result of the study done by Dastgheib et al. (19, 20). The study of Hunt et al.showed that the people who were university educated were are more inclined to use complementary medicine(21). Moreover, Frass et al. showed that the more education was one of the important predictive factors for using complementary and alternative medicine, such a way that the people with higher education used complementay and alternative medicine 1.2 times more than other population (22). These results were in-line with the results of our study.

The results of the present study indicated that the most of the patients were housewives and the least were retired ones. This may be because of that the housewives often have more free time to visit TPM clinics; 
however, the reason for lack of follow up for skin lesions among the retirees may be because of the lower life expectancy and a lack of attention to their appearance. Kaushal et al. demonestrated that the housewives in India used allopathic medicines more than other drugs (23). This result was similar to the result of our study.

The most frequencies of patients referred to TPM clinics in our study were in spring and autumn. This may be resulted from increasing the incidence rate of many skin disorders or aggravition of skin disorders such as atopic dermatitis, eczematous disorders and other inflammatory skin conditions in spring and autumn. In addition, seasonal allergies and skin lesions are more common in these both seasons because of distribution of the pollens in the air $(24,25)$. Furthermore, changing in seasonal temperature and humidity of the climate of maybe the other contributing factors to differnaces distribution of patients to visit the physician because of dermatology conditions.

According to our study, acne was the most dermatologic conditions that the patients had explained in their medical chief chief complaints. Acne is an inflammatory disease with polymorphic lesions including papules, pustules, nodules and cysts that affect the face, chest, and back of the trunk commonly seen in adolescents. According to the previous studies, it is estimated that $9.4 \%$ of the global population are affected by acne, making it the eighth-most prevalent disease worldwide. This condition may affect the pscychological and social aspects of patients with acne (26). Several studies revealed that many of dermatologic conditions are multifactorial disorders. For example, seborrheic dermatitis is significantly associated with the disappearance of normal gastrointestinal flora (27). Moreover, some patients suffering from acne rosacea were found to be infected by Helicobacter pylori (28). In addition, while the exact pathogenesis of sebaceous gland diseases is unknown, some reports suggest that gastrointestinal disorders increase sebaceous gland secretions (29). Our findings showed that the most patients were also suffering from a gastrointestinal problem. Moreover, sleep disturbance, neurological and psychiatric problems were the other common comorbid complaints in patients in our study. In this regard, previose studies confirmed that the dermatological conditions were related to gastrointestinal problems, sleep disterbance, and psychiatric problems (7, 30-33).

There were a few studies around using complementary and alternative medicine in patients with sermatologic conditions. In a study done by Arye et al ., 77 patients who referred to an outpatient dermatology clinic were evaluated in the aspect of using complementary and traditional medicine in treating psoriasis. $62 \%$ used complementary medicine of which $58 \%$ of them receive such treatments from an expert in traditional medicine. Also, according to the study, the use of complementary medicine (mostly traditional medicine) among Arabs was significantly higher compared to Jews. The reason these patients used complementary medicine was they believed that it can increase their quality of life. Some also believed that the side effects and toxicity of herbal remedies were less, and even the usage of complementary medicine reduced their stress (34). All of the patients in our study were visited by one or more dermatologist but they did not have the acceptable therapeutic responses to conventional medicine, so they decided to choose TPM as their last chance. Therefore, since more than $50 \%$ of the patients in our study had moderate and good therapeutic response from TPM, it can be very promising, and it can open wide avenues for further studies.

There were some limitations in our study. First, this was a retrospective study, so for evaluating the exact effects of using TPM in patients with dermatologic conditions, we suggest that the chohort studies should be conducted and the patients are followed up, regularly. Next, some of our patients' document were not complete, so we were faced with few missing data on some issuses, such as the therapeutic response of the patients. Therefore, designing powerful electronic systems can improve studies in this area. Finally, our project was a single-center study, so multi-center studies are recommended for future studies.

\section{Conclusion:}

The findings of the present study showed that a large number of patients with chronic dermatologic condition referred to TPM clinics and acceptable therapeutic responses were seen among some of them. Therefore, development of TPM clinics in dermatology field as a scientific and academic approach can be effective in the treatment of the patients with dermatologic conditions accompanied by traditional medicine. Furtheremor, 
more longitudinal observational studies are recommended to determinate the exact efficacy of treatment with traditional remedies in patients who referred to TPM clinics

\section{Conflict of intrest:}

The authors declare that there are no conflicts of intrest.

\section{Reference}

1. Hay RJ, Johns NE, Williams HC, Bolliger IW, Dellavalle RP, Margolis DJ, et al. The global burden of skin disease in 2010: an analysis of the prevalence and impact of skin conditions. Journal of Investigative Dermatology. 2014;134(6):1527-34.

2. Hahnel E, Lichterfeld A, Blume-Peytavi U, Kottner J. The epidemiology of skin conditions in the aged: a systematic review. Journal of tissue viability. 2017;26(1):20-8.

3. Raikhy S, Gautam S, Kanodia S. Pattern and prevalence of psychiatric disorders among patients attending dermatology OPD. Asian Journal of Psychiatry. 2017;29:85-8.

4. Lee HG, Stull C, Yosipovitch G. Psychiatric disorders and pruritus. Clinics in Dermatology. 2017;35(3):27380 .

5. Evers A, Duller P, Van De Kerkhof P, Van der Valk P, De Jong E, Gerritsen M, et al. The Impact of Chronic Skin Disease on Daily Life (ISDL): a generic and dermatology-specific health instrument. 2008;158(1):101-8.

6. Dixon L, Witcraft S, McCowan N, Brodell RJBJoD. Stress and skin disease quality of life: the moderating role of anxiety sensitivity social concerns. 2018;178(4):951-7.

7. Hamidizadeh N, Ranjbar S, Ghanizadeh A, Parvizi MM, Jafari P, Handjani F. Evaluating prevalence of depression, anxiety and hopelessness in patients with Vitiligo on an Iranian population. Health and Quality of Life Outcomes. 2020;18(1):20.

8. Salman A, Kurt E, Topcuoglu V, Demircay ZJAjocd. Social anxiety and quality of life in vitiligo and acne patients with facial involvement: a cross-sectional controlled study. 2016;17(3):305-11.

9. McGuigan K, McGuigan CJJE-bn. Chronic skin disorders negatively impact women's quality of life and sexual function. 2019:ebnurs-2018-103040.

10. Montazeri A, Sajadian A, Ebrahimi M, Haghighat S, Harirchi IJEjocc. Factors predicting the use of complementary and alternative therapies among cancer patients in Iran. 2007;16(2):144-9.

11. Harirchian MH, Sahraian MA, Hosseinkhani A, Amirzargar NJIjon. Level of attitude toward complementary and alternative medicine among Iranian patients with multiple sclerosis. 2014;13(1):13.

12. Mohammadzadeh-Moghadam H, Kamalinejad M, Nazari M, Khajavi A, Mohammadpour A. Dr. Ahmadieh's Views and Experiences on Melancholy Treatment; a Comprehensive Narrative Review. The Horizon of Medical Sciences. 2015;21(4):43-50.

13. Sehati Shafaie F, Matin Homaei H, Zoodfekr L. Comparison the frequency of menstrual disorders (amenorrhea, oligomenorrhea, dysmenorrhea and premenstrual syndrome) between athletes and non-athletes female students of Tabriz universities, Tabriz, Iran. The Iranian Journal of Obstetrics, Gynecology and Infertility. 2013;16(51):14-21.

14. Kamranpour SB, Farzad L, Rahbar T, Alizadeh S. The effect of valerian on the severity of premenstrual syndrome symptoms. The Iranian Journal of Obstetrics, Gynecology and Infertility. 2015;18(161):1-9.

15. Mousavi P, Zaheri H, Najar S, Afshari P, Hayati F. Effect of vitagnus on premenstrual syndrome. The Iranian Journal of Obstetrics, Gynecology and Infertility. 2015;17(138):1-9. 
16. Reid R, Steel A, Wardle J, Trubody A, Adams J. Complementary medicine use by the Australian population: a critical mixed studies systematic review of utilisation, perceptions and factors associated with use. BMC complementary and alternative medicine. 2016;16(1):176.

17. Mollaoğlu M, Aciyurt A. Use of complementary and alternative medicine among patients with chronic diseases. Acta clinica Croatica. 2013;52(2.):181-8.

18. Jowkar F, Godarzi H, Parvizi MM. Can we consider silymarin as a treatment option for vitiligo? A double-blind controlled randomized clinical trial of phototherapy plus oral Silybum marianum product versus phototherapy alone. Journal of dermatological treatment. 2019.

19. Malekzad F, Rahmati M, Taheri A. Prevalence of Skin Diseases among Nursing Home Patients in Elderly Home Nursings in North of Tehran. Pajoohande. 2007;12(3):253-8.

20. Dastgheib L, Farahangiz S, Adelpour Z, Salehi AJJoe-bc, medicine a. The prevalence of complementary and alternative medicine use among dermatology outpatients in shiraz, Iran. 2017;22(4):731-5.

21. Hunt KJ, Coelho HF, Wider B, Perry R, Hung S, Terry R, et al. Complementary and alternative medicine use in England: results from a national survey. International journal of clinical practice. 2010;64(11):1496502.

22. Frass M, Strassl RP, Friehs H, Müllner M, Kundi M, Kaye AD. Use and acceptance of complementary and alternative medicine among the general population and medical personnel: a systematic review. Ochsner Journal. 2012;12(1):45-56.

23. Kaushal J, Gupta MC, Jindal P, Verma S. Self-medication patterns and drug use behavior in housewives belonging to the middle income group in a city in northern India. Indian journal of community medicine: official publication of Indian association of preventive \& social medicine. 2012;37(1):16.

24. Xu Q, Zhang Y, Zhang L, editors. Effect of perennial dust mites allergy on symptom severity of autumn allergic rhinitis in adults. Allergy \& Asthma Proceedings; 2020.

25. Matvieieva S, Umanets T, Lapshyn V, Haiduchyk H, Antipkin Y. Pollen-food Allergy Syndrome Among Children with Sensitized to Spring Trees. EUREKA: Health Sciences. 2019(2):3-11.

26. Tan J, Bhate KJBJoD. A global perspective on the epidemiology of acne. 2015;172:3-12.

27. Zhang HJCJD. Quantitative studies on normal flora of seborrhoeic dermatitis. 1999;32:399-40.

28. Bhattarai S, Agrawal S, Rijal A, Majhi S, Pradhan B, Dhakal SJKUMJ. The study of prevalence of Helicobacter pylori in patients with acne rosacea. 2012;10(4):49-52.

29. Zhang H, Liao W, Chao W, Chen Q, Zeng H, Wu C, et al. Risk factors for sebaceous gland diseases and their relationship to gastrointestinal dysfunction in Han adolescents. 2008;35(9):555-61.

30. Hawro T, Hawro M, Zalewska-Janowska A, Weller K, Metz M, Maurer M. Pruritus and sleep disturbances in patients with psoriasis. Archives of Dermatological Research. 2020;312(2):103-11.

31. Jørgensen AH, Egeberg A, Gideonsson R, Weinstock L, Thyssen E, Thyssen JJJotEAoD, et al. Rosacea is associated with Helicobacter pylori: a systematic review and meta-analysis. 2017;31(12):2010-5.

32. Pedroni MN, Hirotsu C, Porro AM, Tufik S, Andersen ML. The role of sleep in pemphigus: a review of mechanisms and perspectives. Archives of Dermatological Research. 2017;309(8):659-64.

33. Sampogna F, Picardi A, Chren M-M, Melchi CF, Pasquini P, Masini C, et al. Association between poorer quality of life and psychiatric morbidity in patients with different dermatological conditions. Psychosomatic medicine. 2004;66(4):620-4.

34. Ben-Arye E, Ziv M, Frenkel M, Lavi I, Rosenman DJD. Complementary medicine and psoriasis: linking the patient's outlook with evidence-based medicine. 2003;207(3):302-7. 


\section{Title of the tables:}

Table 1- Epidemiological charstristics of of patients with dermatological condition referring to traditional Persian medicine clinic affiliated to Shiaz University of Medical Sciences.

Table 2- Primary dermatological complaints and comorbid diseases of patients referring to Iranian traditional medicine clinic

Table 1- Epidemiological charstristics of of patients with dermatological condition referring to traditional Persian medicine clinic affiliated to Shiaz University of Medical Sciences.

\begin{tabular}{|c|c|c|}
\hline & Frequency $(\mathrm{N}=361)$ & Percentage \\
\hline \multicolumn{3}{|l|}{ Age Group } \\
\hline Less then 20 & 126 & $20 \%$ \\
\hline 21 to 30 years & 173 & $27.4 \%$ \\
\hline 31 to 40 years & 190 & $30.1 \%$ \\
\hline 41 to 50 years & 72 & $11.4 \%$ \\
\hline 51 to 60 years & 39 & $6.1 \%$ \\
\hline Above 60 years & 31 & $5 \%$ \\
\hline \multicolumn{3}{|l|}{ Sex } \\
\hline Male individuals & 151 & $23.9 \%$ \\
\hline Female individuals & 480 & $76.1 \%$ \\
\hline \multicolumn{3}{|l|}{ Marital Status } \\
\hline Single & 284 & $45 \%$ \\
\hline Married & 347 & $55 \%$ \\
\hline \multicolumn{3}{|l|}{ Educational Stage } \\
\hline Undergraduate education & 146 & $23.1 \%$ \\
\hline Diploma & 107 & $16.9 \%$ \\
\hline Post-diploma & 65 & $10.4 \%$ \\
\hline Bachelor's degree & 214 & $34.2 \%$ \\
\hline Master's degree and higher & 97 & $15.4 \%$ \\
\hline \multicolumn{3}{|l|}{ Occupation } \\
\hline Employees & 151 & $23.9 \%$ \\
\hline Freelance & 56 & $8.9 \%$ \\
\hline Students & 166 & $26.3 \%$ \\
\hline Housekeeper & 187 & $29.6 \%$ \\
\hline Retiress & 18 & $2.9 \%$ \\
\hline Unemployed & 53 & $8.4 \%$ \\
\hline \multicolumn{3}{|l|}{ Month of Visit } \\
\hline Spring & 182 & $28.8 \%$ \\
\hline Summer & 135 & $21.4 \%$ \\
\hline Atumn & 163 & $25.8 \%$ \\
\hline Winter & 151 & $23.9 \%$ \\
\hline \multicolumn{3}{|l|}{ Nationality } \\
\hline Iranian & 626 & $99.5 \%$ \\
\hline Foreign & 3 & $0.5 \%$ \\
\hline \multicolumn{3}{|l|}{ Residence } \\
\hline Shiraz, Capital of Fars provinence & 491 & $77.8 \%$ \\
\hline Other Cities in Fars provinence & 93 & $14.7 \%$ \\
\hline Other provinences & 45 & $7.1 \%$ \\
\hline Foreign countries & 2 & $0.3 \%$ \\
\hline \multicolumn{3}{|l|}{ Type of Refferal } \\
\hline Friends and family & 241 & $88.9 \%$ \\
\hline
\end{tabular}




\begin{tabular}{lll}
\hline & Frequency $(\mathrm{N}=361)$ & Percentage \\
\hline Specialist physician & 2 & $0.7 \%$ \\
Social media & 12 & $4.4 \%$ \\
Own decision & 4 & $1.5 \%$ \\
Unknown & 12 & $4.4 \%$ \\
\hline
\end{tabular}

Table 2- Primary dermatological complaints and comorbid diseases of patients referring to Iranian traditional medicine clinic

\begin{tabular}{lll}
\hline & Frequency $(\mathrm{N}=361)$ & Percent $(\%)$ \\
\hline Primary Skin Complaints & & $27.6 \%$ \\
Acne & 174 & $20.4 \%$ \\
Eczema and Dry skin & 129 & $8.7 \%$ \\
Pruritis & 55 & $5.1 \%$ \\
Urticaria & 31 & $13.5 \%$ \\
Melasma, Vitiligo, Freckles or & 85 & \\
other Pigment disorders & & $13.6 \%$ \\
Hair Loss & 86 & $0.3 \%$ \\
Hirsutism & 2 & $1.9 \%$ \\
Oral Aphthous & 12 & $7.8 \%$ \\
Psoriasis & 49 & $0.3 \%$ \\
Premature Graying of hair & 2 & $0.8 \%$ \\
Nail Disorders & 5 & \\
Comorbid problems & & $47.7 \%$ \\
Gastrointestinal & 301 & $13.5 \%$ \\
Gynecological & 85 & $13.5 \%$ \\
Respiratory & 85 & $27.6 \%$ \\
Neurological and psychiatrics & 174 & $16.6 \%$ \\
Sleep disorders & 105 & $40.7 \%$ \\
Other skin problems (which found & 257 & \\
in history taking) & & \\
\hline
\end{tabular}

